\title{
LA PROTECCIÓN AMBIENTAL Y EL INTERÉS PRIVADO EN EL MARCO INTERNACIONAL. \\ NECESIDAD Y POSIBILIDADES DE UN NUEVO MANDO ORDENADOR JUSTIFICADO.
}

Por Heber Arbuet-Vignali ( $\left.{ }^{*}\right)$ El Pinar - Montevideo.

Noviembre 2002.

\section{Los cambios del siglo XX.}

Quienes hemos nacido hace un tiempo tuvimos el enriquecedor privilegio y la pesada carga de vivir un cambio de civilización de ritmo acelerado. Si bien es cierto que la vida en el planeta es un constante devenir de cambios, hay momentos de la historia en que estos se agolpan y precipitan con vertiginosidad y radicalidad tan grandes que en corto tiempo -el de una vida humana-modifican todas las perspectivas acerca de la totalidad o de la casi totalidad de las cosas; con ello impresionan al ser humano, le desorientan, le conducen a modificar sus puntos de vista y sus escalas de valores, ponen en crisis su fe, le invitan a conocer cada vez mas, le atemorizan con lo que avizora, le asombran, le desconciertan y le obligan a encontrar nuevas explicaciones.

Esto les ocurrió a los seres humanos del Renacimiento y nos ha ocurrido a quienes vivimos desde el promediar del Siglo XX; pero esta vez con una aceleración tal en los cambios de toda naturaleza y calidad que cuando no hemos terminado de comprender $y$ adaptamos a una nueva circunstancia esta ya ha cambiado, resulta obsoleta y debemos comenzar a adecuarnos a otra nueva. El siglo XX ha sido polifacético y caleidoscópico, aunque sus cristales resultaran para el espíritu de brillante luminosidad algunos, profundamente obscuros otros y todos, siempre, estuvieran cubiertos con la pátina gris plomiza de la incertidumbre y la sensación de pérdida irrecuperable de cosas entrañables. 
Una pareja de novios enamorados en 1948 miraría directamente a la Lunallena, como a un fenómeno natural, pleno de encantos y poesía, que conducía los corazones al romance. Esa misma pareja, en 1968, por entonces esposos enamorados y aún tomados de la mano, miraría por televisión, en vivo y en directo, la llegada del ser humano a esa misma Luna ahora llena de posibilidades científicas, constituyéndose en avanzada de nuestra cultura hacia el universo, y colmando de esperanzas y temores a sus corazones.

Las nuevas tecnologías y los adelantos científicos modificaron rápidamente la civilización. Los avances de todas las ciencias naturales y matemáticas, la evolución de los transportes, la facilidad en los desplazamientos y las comunicaciones, acercan a los humanos de todas las culturas, aportan al acervo común de ellos cantidad de nuevos elementos, les pernite llegar al espacio exterior, a los fondos oceánicos, al interior de su propio cuerpo, a los misterios de su genoma. Y a muchas cosas más. Mucho más tardíamente, recién al acercarse el siglo XXI, las ciencias del espíritu, la filosofía, el derecho, la política, la economía, comienzan a sumat sus esfuerzos para aportar orden al progreso y paz a los espíritus que lo llevan a cabo o lo experimentan.

Todo esto produce acelerados y profundos cambios en el ámbito de las relaciones internacionales, que es el que hoy nos ocupa. En poco más de cien años el mudo pasa por los más diferentes sistemas de relaciones internacionales: del equilibrio multipolar con epicentro en Europa, a los enfrentamientos ideológicos generalizados entre democracias, fascismos, comunismos, ideologías dispersantes, totalitarismos y militarismos; del equilibrio bipolar con epicentros no europeos y sus fases de terror y distensión, a la caida del socialismo real y la confusión que le sigue (unipolarismo?, fin de las ideologías?, fin de la historia?, luchá de civilizaciones?, globalización?). Todo ello para arribar a un incierto presente, difícil de estabilizar y concretar, que transita un constante ir y venir entre una Potencia dominante que pretende la hegemonía pero no desea asumir sus costos, una Organización Internacional mundial que oscila entre ser pantalla justificadora o avanzada de una nueva dimensión política, algunos grupos de Estados que intentan integraciones profundas que podrían conducir a lo que yo he llamado un equilibrio multipolar grupal (ver ARBUET. VIGNALI 1993/1, tomo I, Lección II) y la existencia de fuerzas dispersantes, desestructuradoras (transnacionales, terrotistas, mafias internacionales) que pueden conducir al mundo a una instancia de neo feudalización.

Estas circunstancias fácticas inciden en las relaciones internacionales y en el detecho que las regula. Por la multiplicidad y complejidad de los asuntos a considerar, se hace cada vez más dif́cil la interpretación de las relaciones internacionales; constantemente se redistribuyen las fuerzas comprendidas en la ecuación de poder de los Estados; estos se desestructuran, se integran, se fusionan, ise globalizan?, aparecen nuevos, desaparecen otros y se originan nuevas lealtades que algunos llaman post estatales. En este mismo marco se da un nuevo e importante fenómeno: el interés privado empieza a jugar directamente sus cartas en la escena intemacional. Como respuesta el sistema jurídico internacional que encausa todas esas relaciones se esfuerza por estar a la altura de los cambios. En algunos casos se adelantó a ellos como ocurrió cuando se reguló el estatuto 
La protección ambiental y el interés privado en el marco internacional.

Necesidad y posibilidades de un nuevo manto ordenador justificado

del espacio exterior y el de los fondos marítimos y oceánicos; en otros se acompasó a los mismos como se dio con la creación de la Organización de Naciones Unidas y la de las Comunidades Europeas; pero en algunos casos todavia no ha tomado iniciativa. Creemos que esto último ocurre en el tema planteado que considera la aparición directa del interés privado en el plano internacional. Procuraremos brindar nuestra opinión al respecto.

\section{Importancia del derecho en las relaciones humanas.}

Debemos aclarar que en nuestro enfoque sólo nos ocuparemos del derecho positivo vigente aplicable a hechos y espacios determinados. No nos referiremos al derecho filosófico, a aquel que los pensadores pueden crear o descubrir o sienten que les es revelado como producto de sus elucubraciones en búsqueda de las explicaciones últimas sobre los misterios del mundo y de la vida. Nosotros reconocemos la importancia de este último tipo de derecho, lo valoramos en alta estima y tratamos de explicarlo a través de una visión jusnaturalista realista; pero él no es el objeto de estudio, ni de utilidad directa para los propósitos hermenéuticos que hoy nos proponemos porque responde al pensamiento de seres humanos posicionados en determinadas ideas propias de cada uno. Estas posiciones podrán o no compartirse con los demás, pero siempre se tratarán de posicionamientos doctrinarios, que no tiene propósitos prácticos inmediatos, ni afectan, sino indirectamente, la armónica vida en sociedad de seres humanos que coinciden o discrepan pero que, por sobre todo, deben convivir en orden.

Nuestras reflexiones se referirán al derecho positivo y este es sólo un instrumento, pero un instrumento maravillosos e insustituible, para permitir las relaciones humanas en sociedad. El derecho positivo vigente es un acuerdo entre seres humanos que forman parte de una colectividad determinada, que necesitan vivir juntos y que quieren teglar esa convivencia para llevarla adelante de la mejor manera posible. El derecho positivo atiende a necesidades humanas y por lo tanto se adecua a las necesidades humanas.

El ser humano es gregario por naturaleza y necesidad, gusta de vivir en sociedad con sus congéneres y debe hacerlo para apoyarse mutuamente y poder defenderse de grupos ajenos y de la propia naturaleza ante la cual, en solitario, es débil. Por otro lado la propia idiosincrasia irrepetible de cada ser humano hace que cada uno de ellos sienta y valore de manera muy fuerte y profunda su propia individualidad. Con igual intensidad que su impulso antropológico gregario, el ser humano posee impulsos antropológicos dispersantes. Por estas características propias del género, en el mismo momento en que cada ser humano se instala en la sociedad comín y protectora que le satisface, comienza a afirmar su personalidad, a distinguirla, a procurar privilegiarla respecto a los demás y esto crea problemas en sus relaciones que, si no se controlan, pueden llegar a destruir a los otros, a símismo y en definitiva a la sociedad. 
Además de estas condiciones, el ser humano es racional. Tiene capacidad para objetivar su propia conducta y las recíprocas, analizarlas, extraer consecuencias y proponer correctivos. A través de estos procedimientos racionales, los seres humanos que en sus meditaciones trascendentes habían descubierto el derecho filosófico, se reúnen y, asociados, posiblemente a través de la experiencia, crean un instrumento para ordenar bajo un mando conductor justo, o al menos justificado, sus relaciones en sociedad: el derecho positivo.

Con este emprendimiento los seres humanos se organizan; de alguna manera se comprometen a someterse a un mando ordenador que les permita vivir en sociedad sin destruirse recíprocamente; e institucionalizan autoridades y procedimientos que les obligan a hacer las cosas que saben que son buenas y desean hacer, pero que sin esos compromisos no harían y se perjudicarían, aunque luego lo lamentasen. Esto, no otra cosa, ni nada menos, es el derecho positivo vigente: instrumento indispensable para la vida de los seres humanos en sociedad bajo un mando ordenador de autoridades que les sirvan y que se justifican por la legitimación del consentimiento de los mandados, la racionalidad de los mandatos y su necesidad. El devenir del tiempo ha incorporado estas vivencias a la cotidianeidad; ha encarnado la convicción de que para convivir se precisa una autoridad ordenadora que no debe ser arbitraria; y que tiene que estar justificada a través de reglas que la conduzcan a procurar el bienestar común y sean conocidas y aplicadas objetivamente. Es decir, que posean los dos elementos que en algún momento del tránsito del ser humano por la historia se les aparecieron como la esencia del derecho: las ideas de justicia junto a la de certeza y seguridad.

La necesidad de reglas para convivir es ineludible. Por lo tanto ningún emprendimiento que exija necesariamente la convivencia y tienda a mejorarla puede llegar a buen término si no se dispone de reglas claras a través de las cuales encaminarlo. Para funcionar correctamente se requieren reglas claras, justas, ciertas y seguras, es decir, son necesarias las reglas juridicas institucionalizadas. La existencia del derecho positivo se explica por esta necesidad.

Todo sistema de derecho positivo supone la existencia de un mando ordenador justificado. Ese mando ordenador podría responder a la grosería de la fuerza de que dispusiera una autoridad arbitraria o despótica; o a las maquinaciones pérfidas que permitieran a tal autoridad manipular a los ordenados; o a la seducción exotérica de misterios fraguados; pero entonces ese mando no estaría justificado y por ello su naturaleza no sería jurídica; ni tampoco sería duradero como lo indican todas las experiencias históricas.

A lo largo de la historia siempre hubieron normas que ordenaron la vida en sociedad. Estas reglas no siempre fueron jurídicas, pero en la generalidad de los casos estuvieron justificadas. Esto siempre ocurrió cuando se trató de comunidades estables. En estos casos la justificación de la autoridad que ejerce el mando es indispensable y puede provenir de variadas vertientes. En todos los 
Ia protección ambiental y el interés privado en el marco internacional.

Necesidad y posibilidades de un nuevo mando ordenador justificado

casos, siempre, de alguna manera, pero ineludiblemente, el mando deberá concitar la adhesión de la inmensa mayoría de los seres humanos que le están sometidos. La fuerza y justificación del mando jurídico positivo resultará de la voluntad de la inmensa mayoría de sus sometidos. La justificación del mando puede ser la consecuencia de que los mandados reconozcan en quien lo ejerce una habilidad especial para mediar ante la divinidad, o para conducirlos en la guerra, o para proporcionarles bienestar en la paz. También puede responder a la necesidad, por no disponerse de una opción mejor; o a concepciones ideológicas teocráticas o laicas; o a la legitimación de un sistema jurídico. En algunas épocas, la capacidad del jefe para atender necesidades primarias, como proteger de crueles enemigos o alimentar en las hambrunas, pudo, toscamente, justificar o, al menos, explicar el ejercicio del mando. En otros momentos, si la inmensa mayoría de los integrantes de una sociedad coincidían en una ideología teocrática o laica, porque creían en un mismo Dios creador o aceptaban una misma doctrina política, la autoridad religiosa o política en que esa inmensa mayoría coincidian, pudo determinar justificadamente las reglas e indicar a los ejecutores de las mismas. Pero cuando las comunidades se hace más complejas y se fragmentan ideológicamente, la única justificación válida se puede obtener por legitimación a través de un sistema jurídico aceptado por todos o por la inmensa mayoría en base a la doctrina de la soberanía, atributo jurídico que justifica el mando de unos seres humanos sobre otros por legitimación laica, popular y científicamente comprobable (Cfr. ARBUET y BARRIOS, 2001 y ARBUET, 1999).

Cuando la sociedad se agranda, se hace más compleja y se fragmenta, las antiguas vertientes de aceptación y justificación del mando se agotan. Cuando no hay un temor extemo que haga preferible a cualquiera con capacidad de organización suficiente para impedir quedar sometido a ajenos; cuando la libertad de conciencia diversifica las opiniones sobre el más allá; cuando el saber llega a todos los estratos sociales, existe respeto por la opinión del otro y la diversidad sustituye a las políticas monocordes, maniqueas y sectarias; aún entonces y quizá con mayores urgencias que nunca, se torna necesario un mando ordenador y nucleante, que debe encontrarse justificado para lograr sus propósitos. En esas circunstancias, la única justificación del mando se da por la aceptación de los mandados. Este es el principio básico del derecho positivo. La norma está justificaday está bien que se aplique y sea obedecida, porque de alguna manera la inmensa mayoría de los mandados aceptan su autoridad. No la aceptan naturalmente, porque la naturaleza humana no es proclive a ello, pero la aceptan racionalmente y por necesidad.

De la nisma forma en que los seres humanos necesitan del derecho para vivir en sociedad, los conjuntos de seres humanos que se organizan para los mismos fines, las asociaciones, los clubes deportivos, las empresas comerciales y sobre todo la forma más evolucionada de organización política de seres humanos, los Estados, también requieren de normas jurídicas que encaucen racionalmente y con justicia sus mutuas relaciones para ordenar sus conductas y llevar a buen propósito sus objetivos. Esto ocurre, como lo señala con su genial claridad y profundidad KANT (1784 y 1795), especialmente en "Idea de una historia universal desde un punto de vista cosmopolita" y también menos 
directamente en "La paz perpetua"; porque el ser humano trasmite a los entes que crea sus virtudes y sus defectos: su espíritu integrador y dispersante, sus ansias de cooperación y sus impulsos destructivos, su racionalidad, capacidad para superar situaciones y su creatividad.

Por lo tanto las asociaciones de seres humanos y los Estados entre ellas, van a tener los mismos problemas, las mismas posibilidades y las mismas necesidades que los seres humanos que los integran. De dónde, se necesitan las reglas para desarrollar su convivencia. Estas relaciones requieren de reglas muy precisas, muy claras y obligatorias por definición, porque si el interrelacionamiento de los seres humanos es complejo, mucho más lo es el de los conjuntos de seres humanos.

El derecho positivo es una tardía pero perfeccionada forma de ordenar justificada y racionalmente la vida de los seres humanos en sociedad y la de sus creaciones, los Estados, en sus relaciones mutuas.

3. Importancia del derecho en la protección ambiental.

En primer lugar la protección del ambiente humano es un emprendimiento directamente conectado con la vida de los seres humanos en sociedad $y$, como resultado de lo anterior, en esto se encuentra la razón de ser y la necesidad de recurrir al derecho positivo. Además, en el caso del problema ambiental se involucran relaciones humanas interpersonales e institucionales, públicas y privadas, desarrolladas en ámbitos intemos, de jurisdicción común e internacionales. Todo esto contribuye a crear una intrincada red de vínculos y asuntos a encaminar; una compleja situación de cruce de intereses y perspectivas. de dónde resulta imprescindible disponer de reglas claras y técnicamente correctas que procuren soluciones justas y, sobre todo, por su complejo ámbito de producción y aplicación, que proporcionen certeza y seguridad.

En segundo lugar la experiencia histórica reciente muestra con claridad la necesidad de marcos jurídicos correctos e instituciones adecuadas a los fines propuestos para encaminar proyectos internacionales conjuntos sobre temas de exigente prioridad, que involucran grandes intereses de la más variada naturaleza y que sólo pueden ser encausados satisfactoriamente por la coordinación del esfuerzo de todas las partes que someten sus intereses coyunturales propios a metas comunes de mayor satisfacción particular y colectiva y de mayor necesidad.

\section{Las respuestas del derecho a los cambios de la realidad.}

El derecho positivo constituye un instrumento creado en cada oportunidad por los seres humanos para que, obligándolos, reciban de él las ayudas que les permitan convivir en sociedad sin destruirse, individualmente (ámbitos interiores) o en colectividades (relaciones internacionales). Cada sistema jurídico posee algunas bases esenciales inmodificables, ya que de no respetarse se alteraría la 
La protección ambiental y el interés privado en el marco internacional. Necesidad y posibilidades de un nuevo mando ordenador justificado

naturaleza del sistema y sería otra cosa. En consecuencia, en cada caso, dependiendo del problema a reglar, de los sujetos que se involucran y del ámbito espacial en que se ubica, variarán no sólo los contenidos que resultan adecuados para esas normas, sino también las instituciones necesarias y las estructuras jurídicas requeridas para hacerlas funcionar.

El derecho positivo regula y encausa las diferentes necesidades humanas, debe adecuarse a ellas y, en consecuencias, ante distintas necesidades surgirán diferentes respuestas. Con esta perspectiva existen tres marcos normativos posibles identificados y como el último es muy teciente se le debe prestar mucha atención para no incurrir en omisiones, confusiones y errores.

Hasta hace alrededor de medio siglo había dos estructuras jurídicas básicas. Las estructuras de subordinación propias de los sistemas jurídicos internos, enmarcados y con valor dentro de las fronteras nacionales, que se dirigen a conjuntos humanos prácticamente homogéneos, cuya inmensa mayoría de integrantes individuales coinciden, básicamente, en sus orígenes, creencias, escalas de valores, ideas socio políticas y económicas, teniendo fastos y nefastos comunes. Estos factores crean entre ellos una sensación de confianza mutua, por lo cual encuentran satisfactorio vivir reunidos entre si, porque sienten que se asemejan y que estas semejanzas les distinguen de otros conjuntos humanos con los que se relacionan. Estas identidades les permiten someterse a un mando común a través de estructuras de subordinación con autoridades que legislan, juzgan y mandan y sujetos que obedecen. Los sistemas nomativos que establecen estas estructuras se organizan a partir de una dogmática aceptada en la constitución y atienden principalmente a los intereses privados de los seres humanos individuales o asociados (clubes, empresas, etc.), directamente o a través del amparo de intereses públicos al servicio de lo privado individual (organización político administrativa, protección penal, etc.).

La otra estructura existente es la de coordinación, propia del sistema jurídico aplicable a las relaciones intemacionales, con valor fuera o por encima de las fronteras nacionales, en los ámbitos jurídicos no sometidos a soberanía (alta mar, espacio exterior, etc.) oen las relaciones igualitarias entre los soberanos. Este sistema se dirige a colectividades humanas (los Estados) altamente diferenciadas por su origen, tradición histórica, pensamiento filosófico, creencias religiosas, acervos culturales, organización institucional y otros factores (las diferentes "civilizaciones" para algunos autores). Estas colectividades necesitan interrelacionarse y la interdependencia que de ello resulta, hace indispensable la disponibilidad de reglas jurídicas reguladoras. Esto se ha transformado en un imperativo cada vez más profundo, extendido y exigente; pero, como por definición los Estados poseen el atributo de la soberanía, no quieren, ni pueden subordinarse a un mando material externo ajeno a ellos. Todos tienen y quieren defender sus particularidades diferenciales, sin perjuicio de someterse a reglas obligatorias que les ayuden a convivir armónicamente y a cooperar en los emprendimientos que les resultan commes. Estas diferencias e intereses comunes les conduce a reafirmarse 
iguales e independientes y, a la vez, a someterse irremisiblemente a reglas jurídicas de un sistema con estructura de coordinación dónde los mismos sujetos reglados y obligados (los Estados, las Organizaciones Internacionales) son quienes elaboran directamente las normas que les obligan, vigilan su acatamiento y reunidos con sus pares castigan a los infractores. El sistema normativo que establece esta estructura no tiene dogmática y debe justificarse en cada oportunidad, evaluando el interprete todas sus características a través de una hermenéutica jurídica tan rigurosa como especial. Atiende principalmente a los intereses comunes, públicos, de las colectividades humanas que se le someten, protegiendo los intereses privados de sus respectivos integrantes humanos a través del amparo del sistema de vida que ellos aceptan en común y que quieren tener al organizarse en Estados (intangibilidad de las fronteras, respeto a la autodeterminación y supremacía teritorial, protección diplomática, direccionamiento del comercio exterior y el desarrollo interno, defensa nacional, etc..).

Las primeras normas aisladas con estas características aparecieron en el marco de las relaciones diplomáticas alrededor del siglo XV (Cfr. ARBUETVIGNALI, Heber, 1992 y 1993, tomo I, Capítulo I) y se consolidan como sistema general a mediados del Siglo XVII con los tratados de WESTFALIA (Cfr. ARBUET.VIGNALI, Heber, 1993/1, Tomo I, Lección VII, Sección I.). Sus normas poseen gran dinamismo, adaptabilidad y eficacia, agrupándose a lo largo del tiempo en tres grandes modalidades: las de los sistemas clásico, "contemporáneo" y adaptado a la tecnología nuclear.

Por mucho tiempo las tamas del derecho se movieron dentro de esa clara dicotomía. Por un lado estaban las reglas de los sistemas jurídicos internos, cuyas estructuras de subordinación determinan la presencia de autoridades que mandan, crean las reglas, las administran y juzgan a sus infractores y sujetos que deben obedecer a tales reglas y autoridades; estos conjuntos normativos disponen de escasas estrucuras de coordinación dónde el sujeto reglado sea el protagonista en el proceso de creación y puesta en práctica de las normas que le obligan. Por otrolado estaba el sistema del Derecho Internacional Público, en sus diferentes manifestaciones históricas (Derecho Intemacional clásico, "contemporáneo" y el adecuado a la tecnología nuclear. Cfr. ARBUET 1996 y ARBUET 1993/2) que al ordenar jurídicanente a través de mandatos obligatorios a sujetos soberanos o muy estrechamente ligados al atributo de la soberanía, necesariamente posee estructura de coordinación, las que permiten a los propios sujetos reglados y obligados ser los que, directamente, en forma inmediata, creen todas las reglas, vigilen su cumplimiento $\mathrm{y}$, reunidos, sancionen a los infractores.

El primer sistema atiende especialmente las necesidades creadas por las relaciones en que participan directamente las personas privadas, los seres humanos y entes privados y el segundo atiende fundamentalmente a las que resultan del juego de los intereses públicos del conjunto de seres humanos enmarcados dentro de Estados. Por mucho tiempo esta fue una diferencia clara, precisa y única.

Hasta promediar el siglo XX las actividades privadas de importancia poco trascendieron de sus respectivas fronteras nacionales y bastaban para reglarlas los 
La protección ambiental y el interés privado en el marco intemacional. Necesidad y posibilidades de un nuevo mando ordenador justificado sistemas jurídicos internos. Cuando escapaban de ese marco, si no repercutían en los intereses comunes, las ordenaba el Derecho Internacional Privado y si repercutían en forma importante involucrando a los Estados, como en el caso de la protección de los nacionales, las indemnizaciones de guerra, el combate a la piratería, el comercio internacional, la protección de los recursos naturales y otras de similares características, los Estados se involucraban y acomodaban las actividades privadas a través de sus telaciones reguladas por el Derecho Internacional Público. Los seres humanos individuales y los entes no estatales, se conectaban internacionalmente, de alguna manera, a través de sus Estados, pues sólo estos tenían la dimensión y poseían los medios adecuados para realizar las actividades importantes que acaecían regularmente más allá de sus fronteras. En la inmensa mayoría de los casos, directa o, a lo sumo, indirectamente, la actividad internacional de dimensión general pasaba por los Estados y a estos les regulaba un sistema de coordinación, el Derecho Internacional Público.

A partir de mediados del Siglo XX cambian las características apuntadas, se avanza notablemente en la disponibilidad de nuevos recursos tecnologicos; se crean nuevas necesidades y se adoptan nuevas actitudes para satisfacerlas; por estas vías los seres humanos y los entes privados irrumpen y gravitan directamente en las relaciones internacionales, y, en consecuencias, se crearán nuevos tipos de reglas jurídicas para ordenar la nueva realidad. Algunos autores hablan de derecho transnacional, o de derecho supranacional, de un nuevo derecho internacional, o de un derecho "sui géneris" (JESSUP 1967, AKEHURST 1975, FRIEDMAN 1967, PESCATORE 1973).

Se rompe de esta manera la simple dicotomía derecho intemo - Derecho Internacional y se comienza a pensar en nuevos sistemas jurídicos con estructuras mixtas, dónde se equilibran la importancia e incidencia que tienen las estructuras con características de subordinación con aquellas que las poseen de coordinación. En este nuevo marco jurídico deberá ubicarse hoy al derecho destinado a la protección ambiental, pues también en él personas y entes privados han adquirido protagonismo internacional.

Desde que promedia el siglo pasado la realidad nos presenta un doble fenómeno con importante incidencia política y jurídica. Los nuevos medios técnicos multiplican las actividades de interés común para todos los Estados, respecto a las cuales, más allá de todas las diferencias que puedan tener, se imponen las necesidades de la cooperación. Por la misma razón en muchos casos, también inciden directamente en el ámbito internacional y por ende en las relaciones de los Estados, intereses netamente privados, de Organizaciones No gubernamentales, compañáas o individuos, que actúan sólo para si y no para la colectividad estatal de que forman parte. Se abre un nuevo escenario, apatecen nuevos sujetos, las estructuras jurídicas existentes se ven desbordadas en su concepción tradicional; esto complica la convivencia de los seres humanos y, como no alcanza para ordenar la vida el crear nuevas reglas, se revolucionarán los enfoques jurídicos y se procurarán nuevos sistemas con estructuras mixtas que equilibren la subordinación 
y la coordinación, que se ocupen de lo interno y de lo internacional, que amparen los intereses privados atendiendo al individuo, pero que coordinen esto con el amparo de las diferencias que con justicia desean mantener los grupos nacionales organizados en Estados.

La primer manifestación histórica de un fenómeno de esta naturaleza se da en un marco regional al aparecer el Derecho Comunitario europeo (Cfr. ARBUET 1998) que, después de medio siglo, ha asentado sus características esenciales, pero que poco ha sido estudiado desde el ángulo de sus revolucionarias diferencias respecto a las viejas estructuras anteriores. A nuestro entendet, desde las últimas décadas del siglo pasado y para responder a necesidades reales, se están gestando propuestas similares en algunos de los ámbitos de competencia de las relaciones internacionales generales mundiales que involucran a los intereses públicos de los Estados y de las Organizaciones Internacionales intergubernamentales de alcance universal, pero que también y con igual intensidad, afectan a los intereses privados de Organizaciones no gubernamentales, de empresas y de seres humanos individuales. Para nosotros se trata de un notorio fenómeno en plena evolución, pero la práctica de los Estados aún no lo ha tomado en cuenta y sólo parte de la doctrina los percibe, y esto en forma bastante confusa. En este contexto debe ubicarse la protección internacional de los Derechos Humanos, la protección internacional del ambiente humano y otras actividades, en especial económico comerciales; situaciones que consideraremos más adelante.

5. El impacto directo del interés privado en las relaciones intemacionales.

Hasta las últimas décadas del Siglo XX el protagonismo en las relaciones internacionales había pertenecido exclusivamente a los Estados o a estos y a Organizaciones Internacionales creadas e integradas por ellos.

A partir de entonces los intereses privados y quienes los encarnan, intervienen directamente, cada vez con más intensidad, en las relaciones internacionales. Tómese en cuenta que no estoy diciendo que los intereses privados antes no hubieran influido en las relaciones internacionales. Estos intereses siempre existieron y jugaron un papel importante en ese ámbito; pero el cjercicio, la puesta en práctica de estos intereses, la lucha por imponerlos, siempre, o en la casi totalidad de los casos se hacía a través de los Estados. Los intereses privados presionaban dentro de los Estados, lograban el apoyo de la opinión pública o de los grupos políticos dirigentes en sus Estados, para hacer valer desde allí sus intereses en el ámbito internacional. Siempre un Estado actuaba por los privados.

Bastan algunos ejemplos. Cuando en el Siglo XIX un grupo de inversionistas privados europeos vio peligrar sus intereses ante las dificultades planteadas por un gobiemo sudamericano para la devolución de la deuda contraída los Estados cuyos nacionales eran inversores, Alemania, Francia y Gran Bretaña, presionaron al gobiemo de Venezuela para lograr el cobro llegando a realizar un bombardeo conjunto sobre la ciudad de La Guaira.

$\mathrm{Al}$ principio del siglo $\mathrm{XX}$, 
La proteción ambiental y el interés privado en el marco intemacional. Necesidad y posibilidades de un nuevo mando ordenador justificado dos compañías petroleras de las cuales una tenía influencias en Bolivia y la otra en Paraguay, se disputaban la posibilidad de obtener la concesión de la extracción de petróleo en una zona sobre el límite de ambos países y produjeron tantas tensiones sobre sus gobiernos que estos terminaron enfrentados en la Guerra del Chaco. Al promediar el siglo XX el gobierno de Guatemala llevó a cabo una serie de reformas, entre las cuales dispuso la expropiación de tierras de propiedad de la United Fruit Companny, de nacionalidad norteamericana; por la misma época otro gobierno centroamericano dispuso medidas progresistas muy similares sin llegar a afectar los intereses de esa misma compañía. En estas circunstancias, en el ámbito internacional, la presión sobre Guatemala para revertir las expropiaciones no fue hecha directamente por los intereses privados afectados, sino que estos incidieron sobre el gobiemo del Estado de la nacionalidad de la sociedad, los Estado Unidos de América, logrando que este, en el período más duro de la guerra fría, denunciara primero en Naciones Unidas al gobiemo de Guatemala y apoyara luego las acciones de insurrectos intemos tendientes a desestabilizarlo y sustituirlo por otro que anuló las medidas. En este caso y en muchos otros similares los intereses afectados habían sido privados y sus representantes hicieron las gestiones conducentes a adoptar medidas que los ampararan; pero tales gestiones se hicieron en el ámbito interno del Estado de nacionalidad de la compañáa y la actuación en el campo internacional se realizó a través de la diplomacia y la política exterior de ese Estado. Los intereses privados se conmovían por problemas internacionales y procuraban influir en ellos, pero no lo hacian directamente sino a través de la gestión política, diplomática o militar de los Estados.

A mediados del Siglo XX y en Europa, por primera vez se tomó conciencia de que la fuerte presencia del interés privado en los asuntos intemacionales podía conducir a resultados no queridos por los Estados y que ello debía controlarse. Al finalizar la Segunda Guerra Mundial se comprendió que no había sido ajeno a ella, entre otras muchas causas y como lo fuera también en 1870 y 1914 , la puja de los intereses privados de la industria pesada, de la industria de los armamentos, que competían por la cuenca europea del carbón y de acero, que presionaba a sus gobiernos y que se había constiruido en uno de los importantes motivos para los enfrentamientos bélicos en el viejo continente.

Entre las propuestas que hicieron los estadistas que procuraron la recuperación de Europa a través de sus Comunidades, una de ellas encara este problema con verdadera creatividad. Al constituirse la Comunidad Económica del Carbón y del Acero se procuran neutralizar todos los intereses que incidín en uno de los problemas que continuamente desestabilizaban a Europa y para ello se crea una empresa multinacional para atender esos intereses, la que tendrá bajo su control directo a los Estados y a los privados. Se iniciaba el largo proceso que concluirá en la actual Unión Europea.

A partir de la década del $90^{\prime}$ se ha incrementado fuertemente la actividad internacional de los intereses privados, ajenos e independientes de los intereses de sus Estados, en ocasiones indiferentes a ellos, y en otras coincidentes u opuestos. La tecnología al alcance cada vez de más personas pernite este novedoso fenómeno del que nos imponen diatiamente todos los medios de comunicación. 
Esta nueva realidad, impactante desde mediados del siglo XX, puso a disposición de los setes humanos instrumentos que les permitieron incidir en la situación intemacional de otra manera. Lo que ocurre no es nuevo, pero la intensidad y extensión con que se produce sílo es. Desde siempre, o al menos desde que se superóla situación del aislamiento recíproco de los grupos prehistóricos (Cfr. H.ARBUETVIGNALI, 1993/1, Tomo I, capítulo I), los Centros de Poder independientes que se relacionaron to hicieron con frecuencia e intensidad progresivas, resultando por ello cada vez más interdependientes.

En los años más recientes los recursos tecnológicos disponibles permitieron que actividades, que a determinado nivel de producción e incidencia sólo habían podido ser realizadas por los Estados y en algunos casos solo por los grandes Estados, quedarán a disposición de organizaciones privadas, comerciales, financieras o de otra naturaleza, incluso de grupos humanos que escapan o eluden los normales controles estatales, a veces de naturaleza delictivos, $y$, aún, al alcance de seres humanos individuales, actuando por su propia cuenta. Esto que no era novedoso porque siempre había ocurrido en el ámbito interno de los Estados y, en ocasiones, en algunos de ellos, excepcionalmente, con fuertes impactos que les condujeron a crisis de dispersión disolvente, se traslada por primera vez al ámbito internacional y, en ocasiones cada vez más numerosas y gravitantes, adquiriendo un gran impacto en las estructuras tradicionales de las relaciones internacionales. Aparecen en el marco intemacional no sólo las actividades de entes privados persiguiendo fines particulares, sino también los poderes dispersantes, al principio manejados por los Estados como arma para atacar a sus enemigos, rivales o compe tidores y después escapando al control de los mismos. Primero fueron las grandes compañías económico comerciales transnacionales y las organizaciones internacionales no gubernamentales con fines políticos y el propósito de presionar a los Estados; más recientemente se suman a estas fuerzas dispersantes las organizaciones mafiosas onarcotraficantes transfronterizas y los grupos de terroristas con capacidad para conmocionar con sus acciones a los Estados más poderosos y de desestabilizar las relaciones y la economía mundial; a lo que deben agregarse las acciones individuales de quienes manejan medios técnicos que les permiten eludir el control de los Estados

Recordemos, por ejemplo, cuando Francia pensaba realizar una experiencia nuclear en un atolón del Océano Pacífico y una flota de Green Peace se enfrentó a la armada francesa dispuesta impedir tal hecho aún recurriendo a las armas. Green Peace es una $\mathrm{ONG}$, no es un Estado.

Por otra parte el terrorismo internacional, las mafias internacionales, el narcotráfico, los grandes consorcios comerciales internacionales, diatiamente ponen en juego intereses privados que trascienden fronteras y que escapan al contralor de los Estados; que juegan su propia realidad y necesidades en al ámbito directo de las relaciones internacionales. Recordemos al señor, persona física individual, que actuando por cuenta propia, con un ordenador y las técnicas adecuadas desde Londres vació un banco tadicado en Hong Kong; y al señor que en la Argentina, durante mucho tiempo obtuvo que Aerolíneas le pagara un importante sueldo para 
La protección ambiental y el interés privado en el marco intemacional. Necesidad y posibilidades de un nuevo mando ordenador justificado

que con su ordenador no hiciera salir aviones vacíos desde lejanos aeropuertos. Es el caso también del joven alemán que salió de Berlín y aterrizó con un aeroliviano en la Plaza Roja de Moscú, eludiendo todos los sistemas defensivos y conmocionando, en los últimos tiempos de la guerra fría, los equilibrios internacionales. Todas estas son actividades privadas, actuadas directamente por privados, que se realizan en el marco internacional e inciden en él. Es el caso también tan común y con tanta incidencia en estos días de los jóvenes evaluadores del "riesgo país", que nos muestra la televisión, que no se conoce en que marco institucional están actuando y que dicen "tal país tiene tal índice de riesgo" y los capitales se quedan o se van y las crisis se superan o estallan. Como demostración máxima de esta afirmación, recuérdese el atentado contra las Torres Gemelas en Nueva York. Actualmente hay una gran posibilidad de incidencia de los intereses privados directamente en el ámbito internacional, permitida y favorecida por los nuevos medios tecnológicos; y esto hace que en muchos ámbitos de competencia externa no haya certeza ni seguridad pues ni siquiera los Estados más poderosos están en condiciones de ordenar estas acciones. Esto notoriamente complica las posibilidades de concretar una correcta protección internacional del ambiente humano. Las acciones, no sólo del Estado, sino las de una empresa, o las de un individuo, en Piracicaba, al oeste de Sao Pablo, en un extremo de la cuenca del Plata, repercutirán en El Pinar, en el centro sur del Uruguay, situada al otro extremo.

El juego de los intereses privados y de las acciones que ellos determinan, pueden coincidir o no, pueden ser favorables, inocuos o perjudiciales para los intereses y necesidades del común de las gentes y del común de los Estados. Sin normas ni autoridades que regulen estas situaciones imperará el desorden y el perjuicio será para todos. Se hace necesario disponer de un sistema de teglas y de una autoridad ordenadora justificada.

Esta situación repercute en los sistemas jurídicos. Actualmente ha sido superada la dicotomía tradicional entre derecho interno para regular los intereses privados con un sistema de subordinación jurídica y política dependiente del Estado, y Derecho Intemacional Público para regular los intereses comunes de los Estados con un sistema jurídico de coordinación entre Estados soberanos, e iguales dependiente de sus acuerdos. Hoy se dan situaciones fácticas que escapan a toda regulación y control jurídico y político, si es que solo se cuenta para ello con los sistemas clásicos. Ninguno de estos sistemas ha desaparecido ni resulta obsoleto, ni ha perdido eficacia. Son necesarios para continuar aplicándose en su antiguo ámbito de competencias, pero no pueden controlar estas nuevas realidades.

Una respuesta a esta problemática apareció en Europa, para reglar un ámbito determinado de competencias, en principio económicas, con el Derecho Comunitario: un sistema mixto, en el cual se recurre a estructuras de coordinación y de subordinación, que permiten el contralor de los intereses y de las actividades estatales y, a la vez, establecer el contralor, dando respaldo y garantías jurídicas al 
juego de los intereses y de las actividades privadas, que responden exclusivamente a móviles privados.

Después de casi 50 años de práctica, regulación e institucionalización del Derecho Comunitario, lo afirmado es fácil de constatar en el preciso ámbito regional en que funciona. Pero los mismos problemas se dan en las relaciones internacionales generales, a nivel mundial y alli hay muy pocas propuestas doctrinarias de avanzada y los intentos del derecho positivo y de institucionalización son ambiguos e inoperantes al vincularse al sistema del Derecho Internacional Público. A nivel de relaciones internacionales generales no solo no se ha intentado establecer un nuevo sistema adecuado a esta problemática, sino que ni siquiera se tiene conciencia de su necesidad; las propias necesidades generan respuestas que se incluyen en los sistemas tradicionales pero que, en realidad, son esfuerzos para concretar sistemas jurídicos de transición, como se les desconoce, no se ha planteado la posibilidad real de concretarlos, ni se han desarrollado las ventajas de disponerlos.

Creemos que la regulación de varios ámbitos de competencias en el marco de las relaciones internacionales se encuentran en esta situación. El Derecho Internacional de los Derechos Humanos ya no constituye un conjunto normativo ubicado propiamente dentro del Derecho Internacional Público, sino que, al menos, se encamina o pretende establecer un nuevo y diferente sistema en transición. El Derecho Penal Internacional, con la creación de la Corte Penal Internacional de Roma, no puede decirse que continúe siendo estrictamente parte del Derecho Internacional Público; tiene bases en él, se origina en actos del Derecho Internacional Público, pero, una vez que ha sido creado, deja de estar encerrado en este sistema porque, para que funcione, los viejos instrumentos ya no son suficientes. Muchas veces hemos oído mencionar a la nueva "lex mercatore" y se puede comprobar que muchas áreas del comercio internacional, de la economía o de las finanzas internacionales no se regulan ya propiamente por el Derecho Internacional Público y tampoco son alcanzadas exclusivamente por un derecho interno incapaz de cubrir esos ámbitos, o por un Derecho internacional privado que los atienda en todos sus requerimientos desde su perspectiva y posibilidades; hay normas o prácticas que se ubican fuera de estos sistemas.

El ejemplo más típico de esta nueva realidad se concreta con la regulación de la aeronavegación comercial civil internacional que yá no está a cargo del Derecho Internacional Público, ni pertenece al Derecho Internacional Privado, ni tampoco al derecho interno, sino que están regulados por normas de características, en alguna forma, muy originales.

También el tratamiento de los problemas de seguridad y defensa entre los Estados de un proyecto de integración o entre los que actúen cooperando en un marco de Derecho internacional general como el de la Organización de Naciones Unidas procurando establecer un sistema de seguridad colectiva, en algunos de sus desarrollos no están regulados propiamente por el Derecho Internacional Público; 
La protección ambiental y el interés privado en el marco internacional. Necesidad y posibilidades de un nuevo mando ordenador justificado

requieren de nuevas estructuras, de nuevas instituciones, de distintas normas para que el proyecto funcione bien; Con la economía y con el comercio internacionales, está pasando lo mismo.

También la protección del ambiente humano, por las características del objeto protegido y por las actuales posibilidades técnicas de alterarlo que tienen Estados, organismos y personas individuales, exigen nuevas e imaginativas propuestas jurídicas.

Para reglar estas realidades ya no es suficiente el Derecho Internacional Público típico, ni los derechos internos, ni tampoco un tipo de Derecho Comunitario; es necesario un sistema nuevo. No estoy diciendo con esto que el Derecho Internacional Público vaya a desaparecer, él es un instrumento imprescindible para regular un tipo de relaciones entre seres humanos agrupados en Estados y en tanto no haya una coherencia mayor entre las opiniones de las gentes de todo el mundo que permita un gobiemo mundial - y esto puede estar muy lejos de ocurrir - el sistema tiene que seguir funcionando para reglar esos asuntos de la manera en que lo hace actualmente.

Pero hay campos de actividades interestatales que ya tienen otras perspectivas y que para ordenarlas no alcanza con el Derecho Internacional Público puro; y en esta situación creemos que está todo el derecho a la protección ambiental.

Para concretar la protección del ambiente humano va a haber que recurrir a las soluciones del derecho interno, a las del Derecho Internacional Público, a las del Derecho Internacional Privado y también, en algunos aspectos, la regulación habrá de recurrir a nuevos sistemas. Se requerirán solucione jurídicas de Derecho Internacional Público, pues ellas son las adecuadas tanto para concretar los compromisos de los países y Organizaciones Internacionales involucradas, como para incidir en las zonas declaradas patrimonio común de la humanidad o de uso común y libertad. También se requerirán soluciones juridicas de derecho interno de los Estados y del Derecho Internacional Privado para reglar los hechos acaecidos en los ámbitos internos. Pero para la inmensa mayoría de las situaciones que se produzcan, resultará necesaria una reglamentación que resulte de la creación de nuevos sistemas jurídicos, con nuevas estructuras, sujetos, fuentes, formas de relacionarse y fundamento. Ello debe y puede hacerse. Sólo se requieren conocimientos jurídicos, dedicación al trabajo y voluntad política.

6. Necesidad de superar el desorden y la carencia de una autoridad justificada.

Las propuestas jurídicas sistémicas de que se dispone actualmente no son suficientes para controlar esta situación; es necesario procurar nuevos sistemas jurídicos para regular este nuevo tipo de relaciones in ternacionales dónde aparecen otros componentes y actores cuya presencia era escasísima hasta hace poco La respuesta no la puede dar el Derecho Intemacional Público, porque en este, admitir al ser humano individual con sus intereses particulares, como sujeto procesalmente 
activo, determina que el sistema deje de ser un sistema de coordinación; deje de ser lo que es y deje de servir para lo que tiene que servit. Hay que procurar soluciones complementarias como lo que fue en su momento, dentro de su ámbito limitado el Derecho Comunitario. Pero no se solucionará el problema trasladando soluciones regionales al ámbito general, porque aquí los problemas son otros, atnque sean parecidos. Debe diagnosticarse debidamente el caso y luego procurar para él soluciones específicas.

El desarrollo reciente de la presencia del interés privado en las relaciones internacionales, evidencia la carencia de reglas e instituciones adecuadas para su ordenada conducción. Esto es una consecuencia de los hechos. Hasta hace no mucho tiempo, ese control estaba confiado al ámbito interno de los Estados que, en una etapa previa, eran quienes decidian cuales de esos asuntos llevarían al ámbito internacional. De esta forma hasta hace pocos años, la actividad de los agentes privados que actuaban para hacer prevalecer sus intereses en acciones de dimensión intemacional, no se realizaban directamente en ese ámbito, se consolidaban en el ámbito interno para proyectarse de ahí al ámbito intemacional a través de los respectivos Estados los que, bien o mal, con justicia o sin ella, de alguna manera daban certeza y encausaban las situaciones. En defensa de estos intereses podían aparecer Estados que en protección de sus nacionales impusieran reglas injustas, duras para los demás, pero eran dadas en el marco de las relaciones entre Estados y esto determinaba que hubiera una comunidad básica, aunque fuera mínima, de intereses y de estructuras; había tiempos y reglas que se cumplían de alguna manera. Cuando aparecen los privados directamente en la escena internacional, ya no hay estructuras, ni reglas, ni principios que sean de aplicación natural y previsible; los particulares actúan según lo que se le ocurre a cada cual, no hay Estados que bien o mal atiendan al bien común; la regla es: sálvese quién pueda y el que toma más que lo disfrute. Esto no sirve para el común de las gentes; faltan reglas y falta una autoridad ordenadora, falta el control estatal que es lo único que defiende a la comunidad de intereses.

En conclusión, actualmente, en el campo de muchas actividades, las personas e intereses privados accúan directamente en las relaciones internacionales y para controlar esta situación no se dispone de reglas claras, ni de instituciones competentes, ni de autoridades ordenadoras. Es necesario todo esto para que se constituya un mando ordenador justo, o al menos equitativo o, aunque sólo sea, previsible y seguro, dentro de una sociedad internacional profundamente interdependiente, "globalizada", que carece de valores filosóficos comunes y con una gran diversidad cultural que debe respetarse, preservarse y coordinarse. Se nos ocurre que, como en el tránsito de la Edad Media al Renacimiento, nuevamente el problema que se plantea es el de la forma que debe procurarse para un mando ordenador justificado, que permita el desarrollo de una sociedad regulando con coherencia y equidad la relación mando-obediencia, los vínculos entre quienes ponen las reglas, los ordenadores o gobernantes y los gobernados, el común de las 
La protección ambiental y el interés privado en el marco internacional. Necesidad y posibilidades de un nuevo mando ordenador justificado gentes 0 , ahora, el común de los Estados, porque hace 500 años se necesitaba esto para el marco inteno de los Estados y ahora se requiere para el de sus mutuas relaciones, parael marco internacional.

\section{Nuestra propuesta recurriendo a la teorí juridica de la soberanía.}

Las reflexiones que nos hacemos son las siguientes. En primer lugar que, ante la dispersión feudal que en el ámbito intemo de una frontera amparaba los intereses del señot, o de la corporación posponiendo los intereses de lo que BODIN Llama la república, actualmente el Estado, y los del común de las gentes, la respuesta fue la institución de una autoridad ordenadora que sometiera a todos en beneficio del común, lo que se logra a partir de la teoría de la soberanía.

En segundo lugar que después de la Revolución. Francesa y el pensamianto kantiano la teoría de la soberanía fue constituyendo en el soporte de un atributo jurídico legitimante de un poder ordenador dentro de una sociedad encerrada por fronteras, supremo, imprescriptible e irrenunciable, ejercido por uno o varios seres humanos (los gobemantes) sobre el conjunto de ellos (los gobemados), pero que estaba condicionado en su ejercicio.

El mando debe ser único, que es lo que permite ordenar; debe ser supremo ya que de lo contrario no sería eficaz; pero al ser ejercido por seres humanos, ya que no hay otra altemativa en la práctica, debe evitarse que sea arbitrario, razón por la cual ese mando debe estar condicionado en su ejercicio: el gobernante sólo puede desplegarlo para conservar el Estado, no para ponerlo en riesgo, y debe ejercerlo en forma tal que atienda las necesidades y los deseos de los ordenados, que en definitiva son quienes le dan el mando para que haga eso; si se usa de otra manera el gobernante no está legitimado, habrá traicionado el pacto, será arbitrario y se podrá resistir a su opresión.

En tercer lugar, que el contenido de la teoría, fundamento último y real del sistema democrático de gobierno y sustento de los derechos individuales, fue tergiversado y desvirtuado en los siglos XIX y XX por las teorías transpersonalistas de la soberanía del gobernante que la desprestigiaron, a lo que contribuyó también la teoría de la soberanía de los gobemados y las idealistas posiciones de los neo jusnaturalistas.

En cuarto lugar que siendo necesario un orden intemacional para controlar el juego de los intereses privados en ese ámbito, la teoría de la soberanía, en sus distintas dimensiones, puede proporcionar ideas y experiencias que faciliten el establecimiento de normas e instituciones adecuadas para tal fin. Sobre estas bases haremos una rápida reseña de la evolución histórica de la teoría de la soberanía y propondremos algunas reflexiones para su futuro.

Se conoce bajo la denominación "soberanía" a un instrumento de naturaleza plural, fáctica, filosofica, política y jurídica, que constituyó, constituye y 
probablemente continuará constituyendo la síntesis de los conceptos que expresan una idea fuerza ordenadora de las conductas humanas que coinciden en sociedad, bajo un mando supremo que impide a los seres humanos reunidos que destruyan la organización que les comprende y les permita evolucionar y desarrollarse dentro de ella. La teoría siempre tuvo que ver con la adquisición, mantenimiento, gestión y extensión de un poder de mando ordenador ejercido en una sociedad cerrada por uno o más seres humanos sobre el conjunto de ellos. Pero esa idea matriz siempre conservada, se formuló de muchas formas, tuvo distintos anclajes - fáctico, filosófico, político o jurídico-, se propuso fines últimos diferentes y fue transitando hacia la actual concepción que hemos formulado y responde a las conclusiones a que arribamos luego de una prolongada investigación.

En la Edad Media, el poder ordenador de la llamada soberanía o suzeranía, se limitaba a un ámbito competencial dentro de la unidad política (el tribunal, el gremio, la corporación, la ciudad, el feudo), se asemejaba a lo que hoy llamamos jurisdicción, se atribuía a quienes adoptaban la última decisión en un asunto y se explicaba por la tradición consuetudinaria. Era un concepto ubicado en la filosofía.

Con MAQUIAVELO la teoría de la soberanía transita netamente al marco político (Cfr.MAQUIAVELO, 1513, circa y 1520-27, circa y ARBUET-VIGNALI, T.P./1). El gran aporte de MAQUIAVELO es trasladar el ejercicio del poder ordenador de un ámbito limitado de competencias, a otro amplio comprensivo de una comunidad multi integrada (la señonía para MAQUIAVELO, la república para BODIN, el Estado posteriormente). Pero MAQUIAVELO explica el ejercicio de ese poder sólo por el juego de las realidades, lo hace descansar en el mero facto y no procura encontrarle una justificación. En estas condiciones el poder ordenador sólo logra concretarse en forma inestable, transitando un constante conflicto que to hace efímero e incapaz de trascender, manteniéndose o adecuándose, más allá de su circunstancia favorable.

Con BODIN se da el primer quiebre jurídico en la teoría de la soberanía (Cfr. BODIN, 1576.79 y ARBUET-VIGNALI, T.P./2). Por necesidades históricas BODIN hace reposar la soberanía en el monarca absoluto (jefe de Estado que concentra todo el poder) y la extiende a todo el ámbito de la república alcanzando a todos sus integrantes. Se trata del poder único, absoluto y perpetuo que BODIN funda en el Derecho natural, es decir, no en un sistema jurídico positivo, sino en un derecho filosófico, muy emparentado con los fundamentos ideológicos religiosos. Con esta justificación frente a los ordenados, al común de las gentes, BODIN procura dar estabilidad y trascendencia al poder ordenador. El de BODIN es un gran avance, pero el autor queda preso en las necesidades de la época y vincula la teoría de la soberanía con el gobierno absolutista de un monarca y el control de los actos de este sólo a cargo de Dios y su conciencia.

Otro avance significativo en la teoría jurídica de la soberanía lo proporciona ROUSSEAU que procura fundarla en el contrato social, una versión dieciochesca de lo que hoy es la aceptación del mando ordenador por los mandados, los ordenados, 
La protección ambiental y el interés privado en el marco internacional. Necesidad y posibilidades de un nuevo mando ordenador justificado

el común de las gentes; la justificación se procura en una concepción laica, en un derecho positivo y no filosófico (Cfr. ROUSSEAU, 1756,circa). ROUSSEAU radica la soberanía en el pueblo, lo que debilita su concepción porque la torna inestable y tan cambiante que dificulta o impide fundar un orden coherente.

KANT" en su obra "La paz perpetua" lleva a la teoría juridica de la soberanía a su máximo desarrollo anterior a que la misma dispusiera de los instrumentos políticos y jurídicos que permiten un papel protagónico y organizado del común de las gentes (Cfr. KANT, 1784 y 1795 y ARBUET-VIGNALI, 2002). KANT radica la soberanía en la nación, conjunto de seres humanos que trascienden y se perfeccionan en la especie y que está sometido en sus decisiones a reglas preestablecidas. Aunque no lo dice expresamente, la teoría de la soberanía en KANT ya nos presenta un atributo jurídico legitimante de un poder ordenador supremo pero condicionado en su ejercicio al contralor, la voluntad y las necesidades de los mandados.

Estos avances se pierden en el Siglo XIX y buena parte del Siglo XX. Las doctrinas filosóficas y políticas transpersonalistas reviven el absolutismo preconizado por BODIN, pero ahora fuera de época y contexto; vinculan la soberanía con un poder de mando desarreglado, desvirtuando así sus esencias y manipulando sus antecedentes científicos, filosóficos, jurídicos e históricos en provecho de intereses ideológicos perversos.

Una consecuencias de esta incorrecta presentación de la teoría de la soberanía son de las diversas atrocidades cometidas en el siglo XX: guerras, imperialismo, colonialismo, genocidios, violaciones masivas de los derechos humanos, despotismo en gobiernos internos. También lo es la bienintencionada reacción neo jusnaturalista, rescatable como posición filosófica, pero que se equivoca al pretender construir un derecho positivo ajeno a la teoría de la soberanía, la que malinterpreta, acusa de ser la causante de todos los males y pregona las ventajas de su desaparición.

Actualmente, en el marco de nuestra teoría de la soberanía, la presentamos como un atributo jurídico que justifica por legitimación laica, popular y científicamente comprobable, el ejercicio de una especial manera de ejercer un poder ordenador de uno o varios seres humanos sobre el conjunto de aquellos que conviven dentro de una frontera; poder que es supremo pero que está condicionado en su ejercicio a establecer un orden dentro del Estado y a que el mismo sea el que quieren y satisface a los ordenados, al común de las gentes. En el marco interno la soberanía se ejerce como poder ordenador supremo y condicionado en su ejercicio; en el marco exterior la soberanía no se ejerce, sino que se coordina entre soberanos iguales e independientes que crean un sistema jurídico de coordinación y se someten a él para poder tener relaciones ordenadas y fructíferas.

Hasta ahora esto funcionó así y resultó adecuado porque al transcurrir el tiempo, ante los nuevos requerimientos fácticos, el atributo de la soberanía, aún sólo en la práctica y fuera de la teoría, adecuó sus desarrollos a las nuevas 
modalidades persiguiendo siempre el mismo fin: un orden justificado y positivo. Por la misma razón también la protección de los intereses privados en el marco internacional podía ordenarse con el sistema del Derecho Intemacional Público, porque esos intereses no eran protegidos y actuados en el marco de las relaciones internacionales directamente por los privados interesados, sino a través de sus respectivos Estados. Como se trataba de pocos sujetos, actuando en general responsablemente y atendiendo los requerimientos públicos, el sistema era operante y establecía un control racional y previsible de las situaciones.

Por lo que hemos señalado anteriormente, las cosas ya no ocurren de esa manera y se necesita de una autoridad ordenadora de los privados en el ámbito internacional. Esto excede al marco del derecho interno y no es tarea del Derecho Internacional Público, sino que lo será de un nuevo sistema, adecuado para ello, con institutos jurídicos mixtos, de subordinación y coordinación, que se origine en el Derecho Internacional Público y que traiga consigo sus nuevos sujetos, fuentes, principios, fundamento de obligatoriedad, estructuras e institutos. iSerá necesario también instrumentar un nuevo desarrollo de adecuación de la teoría jurídica de la soberanía que justifique el actuar de autoridades ordenadoras supremas en ciertas materias del espacio internacional? Creemos que si y nuestros esfuerzos se encaminan a concretar una respuesta a este desafio. Les pedimos que se sumen a nuestro estudio. Muchas gracias.

Llamada.

(*) Catedrático y Director del instituto de Derecho Internacional Público, Facultad de Derecho de Montevideo, Universidad de la República. Investigador, Premio CONICYT 1999, Nivel III.

Esta obra forma parte de una investigación sobre el atributo de la soberanía, iniciada en 1978 y cuyos resultados vienen siendo adelantados en entregas parciales, entre las que se cuentan las siguientes:

Publicados: La soberanía uruguaya en los espacios marítimos. En Revista Uruguaya de Derecho Internacional, Volumen II, Montevideo 1973, pp. 127 a 155. Algunas reflexiones sobre crisis, realidad y perspectivas del Derecho internacional Público. En IX Curso de Derecho Internacional del Comité Jurídico Interamericano de la OEA -1982-. Ed. Secretaría General de la OEA, Washington 1982, pp. 61 a 104 . El atributo de la soberanía en el origen y desarrollo del Derecho Internacional clásico y contemporáneo y en el actual sistema adecuado a la tecnología nuclear, publicado en Curso de Derecho Intemacional del Comiré Jurídico Interamericano, Washington 1991, en Revista de la Facultad de Derecho, № 5, pp. 21 a 38, Montevideo julio-diciembre 1993, en el Capítulo I de Derecho Internacional Público. Temas de la teoría general, Ed. Facultad de Ciencias Sociales y Jurídicas UNL, Santa Fe, R.A. 1995 y en portugués como O atributo da soberanía 
La protección ambiental y el interés privado en el marco internacional.

Necesidad y posibilidades de un nuevo mando ordenador justificado

en Estudos da Integraçao, $9^{\circ}$ volume, Senado Federal, Brasilia 1996; El concepto de soberanía y el ingreso al MERCOSUR, en Revista Jurídica del Centro de Estudiantes de Derecho, año II, № 5, pp. 61 a 70, Montevideo 1991; De la historia, la filosofía y un nuevo realismo frente a la politica internacional. En Ínter mundo, Revista del Centro de Estudiantes de Relaciones Internacionales, Año I, № 1, pp. 37 a 42, Montevideo 1991. Publicado también en Revista del ejército de la República O. del Uruguay, Año VI, № 9, pp. 53 a 56, Montevideo 1991. La evolución histórica de la relación entre el atributo de la soberanía y las fronteras. En Nuestras fronteras. situación general y problemas. Ed. Fundación de Cultura Universitaria, Montevideo 1992, Tomo 1, pp.15 a 18. MERCOSUR, soberanía y supranacionalidad y sus repercusiones jurídicas y políticas, en Revista de la Integración del Centro de Relaciones Internacionales, año 5 , № 8, pp. 65 a 74, La Plata, Buenos Aires R.A., mayo de 1995; Naturaleza y extensión de la protección internacional de los Derechos Humanos y sus vinculaciones con la soberanía, en Héctor Gros Espiell. Liber Amicorum, volumen I, pp. 21 a 37, Bruselas 1997; Soberanía e integración: conceptos opuestos o complementarios?, en libro colectivo Temas de integraçao con enfoque MERCOSUL, volumen I, ed. LTr, Sao Pablo, Brasil 1997; De fronteras países y soberanías, en Revista Jurídica del Centro de Estudiantes de Derecho, año VI, № 13, Montevideo 1998. La soberanía hacia el siglo XXI: desaparición, divisibilidad o nuevos odres para añejos vinos? en Revista de la Facultad de Derecho $N^{\circ} 15$, Montevideo enero-junio 1999, pp. 93 a 120; El atributo de la soberanía en el ámbito internacional, en El Derecho Digital, primer periódico digital uruguayo. Ed. Wwwelderechodigital.com.uy, Montevideo (Uruguay), diciembre 1999. Evolución de la protección internacional de los Derechos Humanos, en Derechos Humanos. A los 50 años de la Declaración Americana de los Derechos Humanos y de la Declaración Universal de los Derechos Humanos. Libro colectivo. Ed. Universidad Católica y Amalio Fernández, Montevideo 1999. La soberanía, la realidad y la enseñanza del Derecho Internacional, en Jornadas de Derecho Internacional. 18 a 20 de octubre 1999. Montevideo, Uruguay. Libro colectivo editado por Organización de Estados Americanos, Secretaría General, Washington D.C. 2000, pp. 217 a 226. El atributo de la soberanía en el marco de la integración internacional. En libro colectivo: IX Encuentro Internacional de Derecho de América del sur. Los procesos de integración en el nuevo milenio. Universidad Católica de Bolivia. La Paz 2000, pp. 171 a 185. El atributo de la soberanía en Emeric de Vattel. En Revista de la Facultad de Detecho, № 18, julio-diciembre 2000, pp. 165 a 197. Relaciones entre el Derecho Internacional Público y el Privado: soberanía y orden público. En Liber Amicorum en homenaje a Tatiana B. Meakelt. Ed. Universidad Central de Venezuela. Facultad de Ciencias Jurídicas y Políticas. Caracas 2001, Tomo I, pp. 3 a 26. El Estado, la soberanía y el marco internacional. en colaboración con Luis Barrios. En Revista de la Facultad de Derecho №. 20. Julio-diciembre 2001. Montevideo 2002. pp. 11 a 46. La idea de soberanía en el pensamiento Kantiano. En liber amicorum en homenaje a 
Ernesto Rey Caro. Córdoba (Argentina), 2002. Inéditos: El atributo de la soberanía en Juan Kaspar Blunschli (para publicar en Liber Amicorum de Manuel A. Vieira). El atributo de la soberanía en el ámbito internacional (Para publicar en Revista da Associaçao Brasileira de Etudos da Integraçao, Porto Alegre, Brasil y en Seminario en homenaje a Alvaro Bauzá Araujo, Montevideo, 2000). Soberanáa, supranacionalidad y cambios en el sistema interno que exige el ingreso al MERCOSUR (Para Revista de la Universidad Nacional de La Plata, Buenos Aires, Argentina). La idea de soberanía en el pensamiento Kantiano. (Para publicar en Libro de homenaje al Dr. Ernesto Rey Caro. Facultad de Derecho y Ciencias Sociales. Universidad Nacional de Córdoba R.A., 2001). Asilo y refugio. concepto, naturaleza y vínculos con la soberanía. (Para publicar en Cuademo de la Facultad de Derecho, Montevideo 2002). Soberanía, constitución y comunidades. (Para publicar en Cuaderno del Centro Argentino par las Relaciones Internacionales -CARl, Buenos aires 2002). Las exigencias de la civilización en el Siglo XXI y sus desafíos al Derecho de la Integración (Para publicar en Libro colectivo del Primer Acto Académico de la Cátedra Latinoamericana para la Integración. Ed. ALADI, Montevideo 2002). Dimensión jurídica 2002 de los procesos de integración. para disquete del Libro de las Ponencias del V Congreso Euro latinoamericano sobre Integración. Montevideo agosto 2002. Elaborados: La soberanía en El Príncipe de Maquiavelo. La soberanía en la obra de Heber Arbuet-Vignali: I. Artículos entre 1964 y 1966; II. Artículos del lustro 1969-1973; III. Los primeros avances; 1976-1982. Bodín. Primer quiebre jurídico en la teoría de la soberanía. En elaboración: El atributo de la soberanía en Wolfgang Friedmann. El atributo de la soberanía en F.H.Hinsley. El atributo de la soberanía en Jellineck. El atributo de la soberanía en Adolf Waltz .

Referencias de la bibliografia citada.

1. AKENHURST, Michael (1975): Introducción al Derecho Internacional. Ed. Alianza Universidad, Madrid 1975. Primera edición 1971.

2. ARBUET-VIGNALI, Heber (1992/93): Lecciones de Derecho diplomático y consular. 2 volúmenes. Ed. Fundación de cultura Universitaria, Montevideo 1992 y 1993.

3. ARBUET-VIGNALI, Heber (1993/1): Lecciones de Historia de las Relaciones Internacionales. 2 volúmenes. Ed. Fundación de Cultura Universitaria, Montevideo 1993.

4. ARBUET-VIGNALI, Heber (1993/2): El atributo de la soberanía en el origen y desarrollo del Derecho internacional clásico y contempotáneo y en el actual sistema adecuado a la tecnología nuclear. En XVHY Curso de Derecho Intemacional del Comité Jurídico interamericano. Ed. Secretaría General de la OEA, Washington 1993, pp. 39 a 58.

5. ARBUET-VIGNALI, Heber (1996): Las relaciones internacionales y sus reglas. En ARBUET-JMMÉNEZ-PUCEIRO: Derecho Intemacional Público. 5 tomos, Ed. 
La protección ambiental y el interés privado en el marco intermacional.

Necesidad y posibilidades de un nuevo mando ordenador justificado

Fundación de Cultura Universitaria, Montevideo, tomo I, 2edición 1996, Capítulol, Sección I, pp. 17 a 98.

6. ARBUETVIGNALI, Heber (1998): El Derecho Comunitario. En La Ley. Buenos Aires, Año LXII, NOS. 83 del 30 de abril y 87 del 7 de mayo de 1998.

7. ARBUET-VIGNALI, Heber (1999): La soberania hacia el siglo XXI: desaparición, divisibilidad o nuevos odres para añejos vinos?. En Revista de la Facultad de Derecho, Montevideo enero-junio 1999, № 15, pp. 93 a 120.

8. ARBUET.VIGNALI, Hebet (2002): La soberanía en el pensamiento kantiano. En Liber amicorum en homenaje al Prf. Ernesto Rey Caro. Ed. Facultad de Derecho, Universidad Nacional de Córdoba. Córdoba, Rep. Argentina, 2002.

9. ARBUETVIGNALI, Heber (T.P/1): La soberanía en el príncipe de Maquiavelo. En trámite de publicación.

10. ARBUET-VIGNALI, Heber (T.P./2): Jean Bodin: primer quiebre jurídico en la teoría de la soberanía. En trámite de publicación.

11. ARBUETVIGNALI, Heber y BARRIOS, Luis (2001): El Estado, la soberanía y el marco internacional. En Revista de la Facultad de Derecho, Montevideo, juliodiciembre 2001, №20, pp. 11 a 46.

12. BODIN, Jean (1576/79): Los seis libros de la República. Selección, traducción y estudio preliminar de Pedro Bravo Gala. Colección Clásicos del pensamiento № 11. Ed. Técnos, Madrid 1985.

13. FRIEDMAN, Wolfgang (1967): La nueva estructura del Derecho Internacional. Ed. Trillas S.A., México 1967. Primera edición 1964.

14. JESSUP, Phillips C. (1967): Derecho transnacional. Ed. Trillas S.A., México 1967. Primera edición 1956.

15. KANT, Emanuelle (1784): Idea de una historia universal desde el punto de vista cosmopolita. En Dino Passini: Emanuelle Kant. Saggi sulla storia. Ed. Giuffré Editore. Milano 1955.

16. KANT, Emanuelle (1795): La paz perpetua. En Fundamentación de la metafísica de las costumbres. Crítica de la razón práctica. La paz perpetua. Tradución de F.Rivera Pastor. Ed. Porrúas S.A. México 1983.

17. MAQUiaVELO, Nicolás (1513, circa): El Príncipe. Comentado por Cristina de Suecia y Napoleón Bonaparte, con nota preliminar de Guillermo Cabanelas. Editorial Claridad S.A.. Biblioteca Hombres e Ideas, №23, 3i. edición, Buenos Aires 1974.

18. MAQUIAVELO, Nicolás (1520-27, circa): Discurso sobre la primer década de Tito Livio. Traducción e introducción por Ana Martínez Arancón. Alianza Editorial S.A., Madrid 1987.

19. PESCATORE, Pierre (1973): Derecho de la integración, nuevo fenómeno en las relaciones internacionales. Ed. BID.INTAL. Buenos aires 1973.

20. ROUSSEAU, Juan Jacobo (1756, circa): El contrato social. Traducción y estudio preliminar María José Villaverde. Ediciones Altaya. Ed. Tecnos S.A., Barcelona 1993. 